\title{
Emerging ecosystems of information and city: Anglo digital repository
}

\section{SIGRADI2018 TECHNOPOLITICAS \\ xxii congresso da sociedade iberoamericana de gráfica digital 22th conference of the iberoamerican society of digital graphics 07|08|09|novembro|2018 iau usp | são carlos | sp br}

\author{
Marcos Lafluf Cuevas \\ Facultad de Arquitectura, Diseño y Urbanismo | Uruguay | marcoslafluf@fadu.edu.uy \\ Gabriela Barber Sarasola \\ Facultad de Arquitectura, Diseño y Urbanismo | Uruguay | gbarber@fadu.edu.uy \\ Fernando García Amen \\ Facultad de Arquitectura, Diseño y Urbanismo | Uruguay | gbarber@fadu.edu.uy
}

\begin{abstract}
This paper focuses on the implementation of a digital repository and content management of the Museo de la Revolución Industrial, in Fray Bentos, as part of a project carried out in the context of the I+D project "La ciudad inteligente; un palimpsesto digital", currently under development in the Laboratory of Advanced Digital Visualization (Vidialab) of the Faculty of Architecture, Design and Urbanism (FADU). The project main theme is the emerging paradigm of Smart Cities with focus on the territory as an integral cultural landscape. The experience, implementation, processed involved and related topics, are described and analyzed theoretically, in the search of architectural and historical heritage dissemination.
\end{abstract}

Keywords: Heritage; Smart City; TIC; Digital repository; Dissemination of heritage; Free software.

\section{INTRODUCCIÓN}

El desarrollo de proyectos que utilizan TIC para difusión y apropiación del patrimonio cultural se encuentran en una progresiva emergencia con una diversidad de propuestas que abarcan desde la realidad aumentada, realidad virtual, repositorios digitales, exposiciones digitales, instalaciones interactivas, videomapping, software de gestión de contenidos digitales, y un largo etc. que abarca distintas hibridaciones de software, tecnologías y prácticas asociadas a la cultura digital.

En la actualidad, la integración y mediación de las TIC en las formas de vivenciar y relacionarse con el territorio y lo patrimonial, ha replanteado ambas nociones. Lejos de pertenecer a una categoría física y unidimensional, en ambos se incorporan y superponen dimensiones sobrescribiendo múltiples relatos favorecidos por la versatilidad de estas tecnologías. Los replanteos que 1 como marco conceptual y teórico.

La concepción de acceso y reutilización en los términos actuales difiere mucho de los conceptos manejados antes del desarrollo exponencial de las tecnologías de información y comunicación en el presente siglo (sitio web de datos, big data, sistemas expertos, etc.). Los proyectos de difusión centrados en los conceptos referidos reclaman una lectura actualizada 0 contextualizada que pueda integrarse de forma más clara a las nuevas concepciones del territorio y las ciudades.

1 Perspectiva y teoría asociada en sus inicios a Amartya Sen, adoptada por los principales organismos internacionales en la actualidad. interesa abordar en el presente artículo son aquellos que refieren a los cambios en las formas de trabajo sobre la difusión y apropiación de lo patrimonial en el contexto de TIC, así como realizar una lectura que nos permita visualizar el lugar que ocupa éste en el nuevo ecosistema que plantean las smart cities.

Los proyectos destinados a la difusión y revalorización del patrimonio histórico y arquitectónico tienen una gran relevancia en la construcción del territorio, en tanto hacen a la dimensión histórica del territorio y aportan elementos identitarios que lo definen a sí mismo y a quienes lo habitan, así como el acceso y la apropiación de los bienes culturales que éstos implican, ya que juegan un rol en la cohesión social y en el desarrollo cultural, aportando al desarrollo socioeconómico general de la sociedad, tomando la perspectiva del desarrollo humano

En esta línea de justificación y fundamentación se realiza la presentación de este proyecto, para intentar aportar algunas observaciones que puedan ser de utilidad.

\section{EL PROYECTO}

La implementación del repositorio digital Anglo se enmarca en el proyecto I+D "La ciudad inteligente; un palimpsesto digital", actualmente en desarrollo en el Laboratorio de Visualización Digital Avanzada (Vidialab) dependiente del Departamento de Informática aplicada al Diseño (Deplnfo) de la Facultad de Arquitectura, Diseño y Urbanismo (Fadu, UR). Dicho proyecto tiene como motivación la reciente designación del "Paisaje Cultural Industrial Fray Bentos" (también referido como "Anglo") 
como patrimonio de la humanidad por la UNESCO en el año $2015^{2}$.

Este proyecto de investigación presenta varias vetas que involucran las TIC y los nuevos medios destinados a la difusión y la accesibilidad al patrimonio urbanoarquitectónico de formas novedosas ${ }^{3}$. En ese contexto, surge el proyecto Repositorio Digital del ANGLO, para lo cual se forma un equipo multidisciplinario de profesionales de distintas áreas (ciencias de la información, ingeniería, arquitectura, comunicación) para la implementación de este repositorio que agrupa diferentes objetos digitales y documentos de distintos tipos, mediante la utilización de software libre y una serie de modificaciones en el código de éste para manipular mediante los metadatos los contenidos. Información textual, visual, sonora y de valor histórico que fueron digitalizados en el Museo y pueden visualizarse en un mapa donde se disponen las arquitecturas que componen el paisaje industrial del Anglo ${ }^{4}$, reutilizarse en aplicaciones de realidad aumentada u otros desarrollos que referiremos más adelante.

En estos espacios de convergencia que representan estos proyectos, se presentan una serie de cuestiones que se pretenden observar y problematizar a partir del estudio del caso mencionado, con el enfoque en tres ejes:

a. el ecosistema informacional y de comunicación en el que se desarrollan estos proyectos (universo de metadatos, interoperabilidad, acceso y reutilización).

b. el potencial del código abierto o software libre.

c. Los elementos vinculantes con la ciudad desde las concepciones de la smart city.

\section{METODOLOGÍA}

Se realiza una descripción general del proyecto, concentrando el análisis e interpretación en los elementos que nos permiten indagar las cuestiones de interés señaladas anteriormente y compartir con la comunidad la experiencia transitada para aportar a los debates implicados.

La descripción general del proyecto se estructura teniendo en cuenta los principales componentes que

2 El Paisaje Industrial Fray Bentos se ubica en el Departamento de Río Negro (Uruguay) y abarca 275 hectáreas que incluyen la arquitectura del frigorífico Liebig's-Anglo, sus instalaciones industriales, los muelles sobre el río Uruguay, el matadero, las áreas dedicadas al pastoreo, las residencias de jefes y obreros y sus lugares de esparcimiento. Su valor radica en que el lugar permite " $(.$.$) aprehender la totalidad del proceso de una$ producción de carne que tuvo importancia mundial". Extraído de documento Paisaje Industrial ANGLO presentado a Unesco, elaborado por IMRN (2015).

3 Para mayor profundización en estas formas novedosas de interacción con el patrimonio que plantea el proyecto Palimpsesto Digital, ver trabajo "Interactive Projection Mapping: Proyecto Patrimonio ANGLO" Barber;Lafluf (2016) donde se describe y presenta la utilización de realidad aumentada proyectada para acceso a información del patrimonio en cuestión.

4 Acceder a http://fraybentos.patrimonioanglo.com para explorar repositorio. hacen al proyecto. A motivos de claridad en la exposición, se divide en tres módulos:

1) Software libre; 2) Trabajo con metadatos y 3) Productos o desarrollos a partir de gestión de contenidos digitales mediante software (aplicaciones, exposiciones virtuales, visualización de información, reutilización para realidad aumentada, etc.).

Concentrándose en la descripción y análisis del software libre y su relevancia en el proyecto, el ecosistema informacional en el que se inscriben teniendo como referencia tres conceptos: el acceso, la reutilización y la interoperabilidad; nos permite por esta vía aproximarnos a las implicancias de estas herramientas en la difusión y apropiación del patrimonio en la ciudad en contexto de TIC.

Las conversaciones teóricas que guían el análisis del caso de estudio referido lo integran las lecturas actuales sobre el patrimonio en el contexto digital y smart city, así como los estudios del ámbito de la ciencia de la información referidos a software libre, repositorios digitales, metadatos y tratamiento de objetos culturales.

\section{EL REPOSITORIO}

Los repositorios digitales son colecciones de objetos digitales de diferente naturaleza (textos, imágenes, sonido), que ofrecen un mecanismo para depositar material por parte del creador, el dueño y el administrador. Cuentan con una arquitectura que maneja tanto el contenido como los metadatos, ofreciendo servicios básicos tales como búsqueda y recuperación, administración, controles de acceso y permisos (Heery y Anderson, 2005).

Entendemos que los repositorios digitales son una materialización del fenómeno de desarrollo del software libre, dónde aplicaciones de código abierto como Dspace, Fedora u Omeka ${ }^{5}$ entre otros han colaborado para que se produzca un progresivo aumento de ellos en el contexto nacional e internacional. Las implicancias de ello trascienden el ámbito de la difusión del patrimonio y nos conecta también con los conceptos de ciudad inteligente, en la medida que estos software gestionan contenidos mediante metadatos que pueden ser integrados a otras plataformas que hacen a la dimensión informacional de la ciudad.

\footnotetext{
5 Dspace: software de código abierto, desarrollado en alianza entre el MIT y HP, que provee herramientas para la administración de colecciones digitales. Fedora: (Flexible Extensible Digital Object Repository Architecture). Omeka: Los responsables de Omeka la definen como una plataforma de publicación web libre, flexible y de código abierto, pensada para mostrar colecciones digitales y exposiciones virtuales de bibliotecas, archivos y museos. Se trata de un proyecto del Roy Rosenzweig Center for History and New media, responsables también del gestor bibliográfico Zotero. Omeka se encuentra liberado bajo una Licencia de software libre (GPLv3), con lo cual su distribución, uso y modificación son libres.
} 


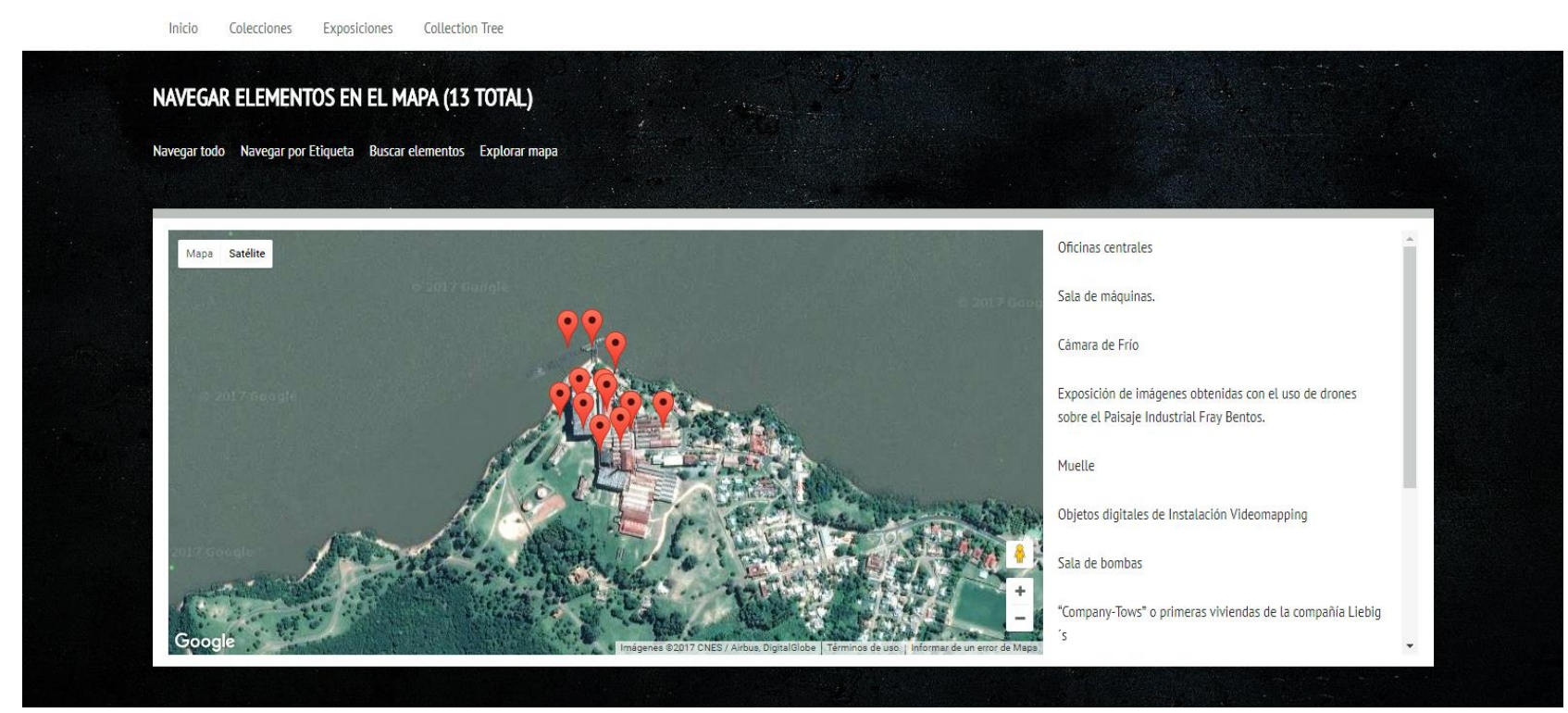

Figura 1: Captura de pantalla de utilización de mapa y georreferencia de documentos. Fuente: Autores.

Si bien el software puede entenderse como un elemento fundamental, que hace al repositorio digital, a motivos de este artículo nos concentrarnos en dos de estos elementos interrelacionados, los metadatos y el software, en tanto son ellos los que nos permiten observar y reflexionar sobre la difusión y otros puntos referidos. Estos son entendidos como herramientas para la construcción de ciudadanía, permitiendo la participación en los procesos de significación y resignificación del patrimonio arquitectónico y cultural de la comunidad.

En este sentido, entendemos que construir un repositorio digital es construir territorio en su dimensión cultural e informacional. Se entiende que aportan a la conformación de espacios digitales en la ciudad de Fray Bentos, potenciando y dinamizando los procesos que hacen al desarrollo cultural, ya sea, brindado material para el desarrollo de objetos educativos digitales así como posibilitando su recuperación y reutilización para otros fines.

Para la creación del repositorio se ha implementado y adaptado el software libre Omeka, tanto para agrupar como para gestionar los contenidos digitales del Museo de la Revolución Industrial. La gestión tiene como motivo, la difusión y el acceso a documentos visuales de formas novedosas (acceso mediante mapa, galerías digitales georeferenciadas). Al momento se han agrupado en el repositorio 200 documentos aproximadamente, entre fotografías, mapas, postales, planimetrías y otros documentos. Conjuntamente se generaron 50 exposiciones digitales georreferenciadas respetando la estructura programática arquitectónica, que agrupan distintos documentos afines con temáticas de interés general (historia, orígenes, evolución).

\section{MÓDULO 1. SOFTWARE LIBRE: BREVE DESCRIPCIÓN Y ANÁLISIS}

Para el desarrollo del repositorio se optó por el software libre Omeka, según SOARIN (2011), este software se encuentra en la intersección entre un gestor de contenidos web de propósitos generales como Wordpress - Drupal, los software de repositorio como Dspace,
Fedora y los sistemas de gestión de colecciones de los museos como Domus o TMS.

En este sentido Omeka se puede entender como un software libre para generación de repositorios y gestión de sus contenidos digitales utilizando estándares internacionales de intercambio de datos de W3C, esquemas de marcado XML y RDS, admitiendo la utilización de estándar de metadatos genéricos, por ejemplo, Dublin Core o específicos como VRA CORE (Visual Resources Association Core Categories) y el protocolo OAI-PMH ${ }^{6}$. Siendo un software no propietario, de código abierto, la participación de un ingeniero en programación habilitó la personalización de ciertos aspectos que permitieron adaptarse a las peculiaridades del patrimonio objeto del proyecto.

La hibridación propia de los software culturales que refiere Manovich (2003) se puede observar de forma clara en el software utilizado en el proyecto. Por un lado, las exhibiciones promueven la reutilización y potencian las cualidades de comunicación de los objetos digitales, ya que existe un proceso de selección y narración de los objetos digitales. Por otro lado, la gran variedad de plugins, su carácter de software libre y abierto son cualidades a destacar ya que promueve distintos usos: adaptabilidad, accesibilidad e interoperabilidad a través de protocolos y metadatos de fácil uso.

\section{EL CÓDIGO ABIERTO Y LA DIFUSIÓN DEL PATRIMONIO. OPORTUNIDADES E IMPLICANCIAS.}

Como primera observación a destacar, estamos frente a un proyecto para y propio de un museo, el Museo de la Revolución Industrial, pero realizado por un grupo foráneo a este. Lo cual es sintomático de un fenómeno generalizado asociado al desarrollo de las TIC. EI traslado y apertura de actividades y proyectos relacionados al patrimonio que anteriormente estaban circunscritos a determinados ámbitos asociados a instituciones de la memoria como museos, comienzan a

6 Open Archive Initiative-Protocol for Metadata Harvesting (OAI$\mathrm{PMH})$. 
ser abordados y realizados desde otros ámbitos donde convergen distintos colectivos 0 entes institucionales (facultades, empresas, colectivos culturales, etc.). De esta forma, distintos profesionales de variadas áreas (es el caso de arquitectos, documentalistas, ingenieros, diseñadores entre otros) son agrupados a partir de proyectos comunes trabajando de forma interdisciplinar $y$ transdisciplinar, trasladando y combinando diferentes formas de hacer.

Estos traslados y aperturas relacionadas estrechamente con este tipo de proyectos, se dan en una intersección donde convergen una progresiva digitalización y un gran desarrollo de software para gestión de metadatos con código abierto, lo que ha derivado en mayor accesibilidad a herramientas para la difusión y revalorización del patrimonio, habilitando la participación de nuevos actores en los procesos.

Una actividad específica en la que los museos llevan varios años trabajando, es la descripción o catalogación de objetos culturales y desarrollo de metadatos (entendiendo la ficha catalográfica como una antigua estructura de metadatos dirigida a recoger datos para el acceso a estos). Desde la revolución de las TIC, esta exclusividad se ve modificada, y es intervenida desde varias áreas, como es la ingeniería (codificaciones, esquemas XML, software de gestión de metadatos), el diseño (interfaz de acceso) y otras ramas de las ciencias de la información abocadas al desarrollo de normativas y estructuras de metadatos para la descripción y recuperación de datos de objetos arquitectónicos u artísticos.

En el panorama actual, con la expansión y accesibilidad del software libre, es posible que un grupo de docentes o un colectivo minoritario pueda realizar su propio sistema de información sobre un objeto o patrimonio determinado e implementar una galería o una exposición digital, insertar los objetos culturales y su información en los canales de información que circulan en la ciudad, así como en el ámbito global, interviniendo en los flujos de información que se despliegan sobre las ciudades y los territorios, sin tener requerimiento de gastos en infraestructura o licencias de software.

\section{MÓDULO 2. TRABAJANDO CON METADATOS}

Podemos definir a los metadatos como los datos que describen las características de un recurso. Se utilizan, ya sea por personas o computadoras, para varias funciones, por ejemplo, localización, búsqueda, descripción, evaluación y selección. Existen distintos tipos de metadatos, ya que se desarrollan en distintos ámbitos de trabajo. De igual manera, en estos últimos años, se han desarrollado varios estándares que contribuyen a su normalización. Dichas normas se elaboran a través de acuerdos generales de los especialistas o grupos de trabajo de cada área o disciplina en la que se utilizan.

En el marco de la implementación de un repositorio, y por lo antes mencionado, se entiende necesario realizar un estudio de los diferentes sets de metadatos y determinar cuál es el más adecuado para aplicar a las diferentes colecciones de fotos, mapas y archivos que conformarán el repositorio.
En la línea de López, Sánchez, Pérez (2003) es posible considerar que el material audiovisual, si bien comparte algunos atributos con el material textual, demuestra cierta complejidad en los procesos de producción, edición, difusión y archivo; que representan variables capaces de dificultar su recuperación. Es necesario estudiar esquemas de descripción que garanticen la gestión y distribución de este tipo de materiales, así como evaluar en qué medida los estándares de metadatos utilizados aproximan al usuario al contenido de un recurso (CalderaSerrano y Freire-Andino, 2016).

La opción tomada en una primera etapa es la semántica del Dublin Core $^{7}$, el cual como se refiere en Grupo de estudio metadatos repositorio Colibri (2014), ha sido establecida por un grupo internacional e interdisciplinario de profesionales de biblioteconomía, la informática, la codificación textual, la comunidad museística, y otros campos teórico-prácticos relacionados. Su uso se generalizó y en el año 2003 se convirtió en norma ISO $15836 / 2003$.

Se profundizó y utilizó dicho estándar de metadatos para el presente proyecto. Esta opción se fundamentó en los siguientes criterios: a) Es el más utilizado en los repositorios regionales e internacionales y ante la posibilidad de integrarse a estos en el futuro, es necesaria la compatibilidad de estándar. b) Cumple con los requerimientos técnicos, administrativos y de preservación. c) Presenta facilidad en su uso. d) Es el estándar elegido por el protocolo de interoperabilidad OAI-PMH. e) Puede ser utilizado con el software Omeka.

De todas formas se pretende seguir explorando, en una etapa posterior, otro estándar de metadatos compatible con el software Omeka ${ }^{8}$, manejado como opcional de sumo interés para la difusión de información en el ámbito de la arquitectura, como es la estructura VRA CORE (Visual Resources Association Core Categories). Según la Library of Congress Cataloging Publication Datapage (2010, p. 24) "el método adecuado, para describir la documentación visual del patrimonio cultural y de los objetos de museos."

\section{METADATOS Y CULTURA EN LA SMART CITY}

Como señala Bonete Vizcaino (2016) es generalizada la ausencia en la profundización de la dimensión cultural de las smart cities, siendo el patrimonio cultural el gran olvidado en los estudios o catálogos de los proyectos asociados a smart city. Si bien como señalan Sanches y Bonete (2016) estos se conectan con varios sectores como es el turismo, el urbanismo o la arquitectura, son una fuente de riqueza para el desarrollo de las ciudades. Visto esto en el contexto de ciudades en proceso de desarrollo en países con un gran ingreso por turismo, resultaría paradójico no detenerse en este punto. Teniendo en cuenta los límites del presente trabajo, está en la intención del mismo hacer visibles algunos axiomas que conectan estos proyectos con el concepto de smart city.

7 http://www.dublincore.org

8 El reciente plugin de Omeka VRA CORE permite el manejo de este estándar de metadatos. Disponible en http://omeka.org/codex/Plugins/VraCoreElementSet 
Esta referencia nos mantiene atentos a la hora de abordar las concepciones duras y cerradas de smart city, entendiendo como muchos autores la ambigüedad implícita de este y su condición de término presentativo más que representativo de una realidad en construcción.

Sin duda remite a un uso óptimo de la información, interconectada y disponible en la actualidad para comprender mejor, controlar sus operaciones y optimizar el uso de recursos limitados (IBM,2011).

Como refiere organismo de Medelín ${ }^{9}$ en su web, una smart city se distingue de otras por una mayor disponibilidad de información, herramientas y capacidad de los ciudadanos de mejorar su entorno, entre otros elementos ya referidos. En este sentido, se pone a disposición material sobre el patrimonio en su gran mayoría bajo licencias creative common para su posterior reutilización, y se genera un cúmulo de datos (metadatos) que pueden ser recogidos por otros sistemas de información (Protocolo OPM) de forma automática, o que permitan el diseño modular que presente la arquitectura del sistema de información, pueden ser utilizados para el diseño de objetos digitales educativos así como permitir la integración, utilización y reutilización de los metadatos y datos por otras aplicaciones, como aquellas relacionadas a realidad aumentada orientadas al museo. Es así que conforman aportes relevantes para la construcción de ciudades más inteligentes.

Como señala Komminos et al (2012) una de las ventajas de la revolución de los datos en las ciudades, es la posibilidad de gastar menos, en tanto el código abierto está disponible, así como la posibilidad de reutilizar datos recogidos en otros sistemas, siendo clave para cualquier estrategia de una ciudad inteligente, el software libre. En este sentido, este proyecto asociado a lo cultural y patrimonial, materializa esas premisas y hace observable esa situación, en tanto el proyecto se ve favorecido y se torna realizable en la medida que es posible prescindir de gastar en licencias de uso, por la existencia de software y códigos abiertos (Omeka). Por otro lado, se desarrolla un cúmulo de datos que es posible compartir y poner a disposición de toda la sociedad para posibilitar un mayor dinamismo y desarrollo cultural de la ciudad.

Según Campbell (2012) y Hollands (2008), se encuentra con distintas asociaciones, un concepto de interés: la idea de centralizar la información. Desde los distintos enfoques posibles, la gestión de la información es fundamental

Fray Bentos, la ciudad en la que se inscribe el patrimonio sobre el que se trabaja, se contextualiza en un país con alto desarrollo en lo que refiere a datos abiertos, gobierno electrónico, acceso y uso de internet, y por sobre todo está casi completamente cableado con fibra óptica y dispone de cobertura $4 \mathrm{G}$ en casi la totalidad del territorio.

Se podría entender esto como un contexto principio para hablar de smart city, o por lo menos de las implicancias en la dimensión cultural asociados a este concepto, como es brindar un sistema con base tecnológica que permita mayor acceso y difusión del patrimonio cultural a través

9 Web disponible en http://www.integramun.org/integramun/ del uso intensivo de TIC como refiere Bonete Vizcaino (2016).

\section{MÓDULO 3. PRODUCTOS Y DESARROLLOS A PARTIR DE LA GESTIÓN DE CONTENIDOS DIGITALES MEDIANTE SOFTWARE. GESTIÓN Y DESARROLLO DE SUBPRODUCTOS A PARTIR DEL TRABAJO CON SOFTWARE Y METADATOS.}

Una breve descripción de este módulo, nos permite concluir la visualización de esos procesos de interacción y la construcción del territorio por medio del uso de las nuevas tecnologías de la información.

Generación de colecciones y subcolecciones digitales de contenidos del Museo de la Revolución estructurados de tal forma que representen la estructura física del Frigorífico y el paisaje industrial en general. La posibilidad de generar un árbol de colecciones mediante el software ${ }^{10}$, permite que los visitantes del repositorio en su proceso de navegación vayan tomando una idea general del paisaje y los espacios implicados.

El proyecto contempla para una segunda instancia hacer uso intensivo de la base de datos obtenida, y los potenciales del software, para el desarrollo de lo que podemos llamar subproductos

a) Explotará la construcción modular y la posibilidad de descarga que habilita el software así como el uso de licencias creative common para propiciar y posibilitar la reutilización de contenidos para la elaboración de objetos de aprendizaje y ODEs (objetos educativos digitales) en el ámbito de la educación primaria y secundaria, en tanto muchos contenidos se asocian a los programas curriculares de estos.

b) Realidad aumentada en el paisaje industrial reutilizando los contenidos del mismo repositorio, para mayor conocimiento y apropiación de quién visita el lugar.

c) Aplicaciones y turismo. Se prevé para una etapa más avanzada del proyecto, enlazar el repositorio a aplicaciones relacionadas al sector de turismo en Uruguay como una forma de colaborar a la visualización y posicionamiento del Paisaje Industrial del Anglo como un destino turístico en el interior del país.

\section{NUEVO ECOSISTEMA DE INFORMACIÓN Y COMUNICACIÓN: NUEVAS FORMAS DE DIFUNDIR EL PATRIMONIO}

Una premisa que se intenta explorar con la descripción y análisis del presente proyecto es aquella que resumimos en la siguiente ecuación: un nuevo ecosistema de información y comunicación es igual a nuevas formas de hacer difusión del patrimonio.

Desde una perspectiva general y teórica podemos decir que el ecosistema informacional ${ }^{11}$ donde se instalan estos

10 Plugin Tree (http://omeka.org/add-ons/plugins/collection-tree/)

11 Tomando el concepto teórico introducido por Neil Postman en el año 1968, el cual aborda a los nuevos medios a partir del uso de la metáfora ecológica como representación del contexto en el que se utiliza dicho medio.
5 
proyectos se caracteriza por la convergencia e hibridación de distintos software y tecnologías y prácticas en cuyo seno se produce un remix profundo, teniendo presente los planteos de Manovich (2008). Estos procesos de hibridación y convergencia que caracterizan al ecosistema, se ven favorecidos en gran medida por la naturaleza digital de los medios que la habitan. A grandes rasgos esta naturaleza digital se puede caracterizar a partir de cinco elementos, tomando al mismo autor, representación numérica o digital (codificación binaria, que permite la recodificación, lo que implica facilidad de manipulación), modularidad (se conforman a partir de varios objetos mediáticos), la automatización (procesos automatizados mediante software), la variabilidad (circula en varios medios) y transcodificación (cultural e informática). Si tomamos el proyecto específico que presentamos y nos detenemos en el software y los procesos que se realizan, se puede observar claramente cómo se puede representar 0 asociar con estos elementos referidos por Manovich (2003).

Específicamente en lo que refiere a los circuitos de información de patrimonio cultural, arquitectónico e histórico y sus formas de difundir, al observar otros casos y teniendo en cuenta los estudios especializados al respecto de repositorios digitales o banco de datos relacionados a patrimonios, podemos destacar por un lado que se caracterizan por un gran desarrollo del trabajo con metadatos, con el desarrollo de varios estándares internacionales de descripción o catalogación de objetos culturales, cada vez más específicos según el tipo de objeto que se describe, como una manera de extraer la mayor cantidad de datos pertinentes e identificadores para permitir una mayor manipulación por el software de gestión de metadatos u otros códigos, así como permitir el intercambio de información con otros sistemas de información de la red.

Otros aspectos que hacen a este ecosistema en relación al patrimonio y a los repositorios, es la utilización de software que trabaja con $\mathrm{xml} u$ otros lenguajes que permiten el acceso y utilización desde móviles u otros sistemas y permiten su reutilización desde otras aplicaciones. De alguna manera esto nos conecta con la comunicación M+M (máquina con máquina) que ha permitido expandir la red de información digital que hace a la dimensión informacional de las ciudades y los territorios, entendido como un componente que hace a la ciudad inteligente.

Siguiendo con esta comunicación máquina-máquina y la automatización de procesos implicada, la utilización del protocolo OAI-PMH por parte de software como Omeka favorecen el surgimiento del fenómeno de los agregadores de contenidos. Un claro representante de esto en el ámbito del patrimonio cultural, es Europeana ${ }^{12}$, estos actúan como grandes bancos de datos que se alimentan de otros sistemas que se han abierto para la cosecha de sus metadatos mediante este tipo de protocolos. En este sentido, los flujos de información que hacen a la imagen cultural y el patrimonio en general tienen en su dirección un condicionante cibernético, que

12 Disponible en http://www.europeana.eu/portal/es transforma los conceptos de accesibilidad, visibilidad y difusión de la información ${ }^{13}$.

A partir de esta breve caracterización del ecosistema, podemos referir a tres elementos que se presentan en estos ecosistemas y que funcionan como ejes fundamentales del proyecto ayudando al presente abordaje: el acceso, la interoperabilidad ${ }^{14}$ y la reutilización.

Cuando nos referimos a accesibilidad, el concepto que interesa es el que hace referencia a la disponibilidad de los recursos para su acceso, descarga, copia, distribución, impresión, enlazado de sus metadatos. El elemento diferencial respecto a los sistemas convencionales, es que los metadatos están normalizados y son recolectables a través de procesos automatizados, facilitando su reutilización sin ninguna barrera financiera, técnica o semántica. (Cerdá, 2015). En este concepto se agregan elementos a la definición desarrollada inicialmente por declaración de la "Budapest Open Access Initiative"15, que nos permiten visualizar lo que referimos como acceso en términos de smart city, donde la interoperabilidad de los sistemas es fundamental no sólo para garantizar el acceso a las personas desde varias tecnologías distintas, un celular, una tablet o pc, sino que otros sistemas -máquinas puedan acceder y reutilizar los datos para otras finalidades.

La perspectiva (tecnócrata) de smart city es insistente sobre la efectividad perseguir a partir de la gestión de la información en las operativas más básicas de los sistemas que hacen a una ciudad (transporte, turismo, seguridad, etc.) y su desarrollo económico y sociocultural. La efectividad en la operatividad y desarrollo de cualquier sistema tiene como requisito el flujo constante de información en forma bidireccional y circular para permitir los procesos de retroalimentación que garantizan su constante auto-regulación y funcionamiento (retomando el origen cibernético de este tipo de perspectiva o relatos que en su forma pura retrotraen a la cibernética de Norber Weiner de los años cuarenta).

El ecosistema de información de lo patrimonial referido anteriormente replica estas lógicas y funcionamientos hoy asociados a las smart cities. A modo de ejemplo, no es sustentable que una aplicación orientada al turismo de la misma ciudad vuelva a digitalizar, describir (metadatos) y conformar una base de contenidos digitales referidos al Patrimonio Anglo cuando puede reutilizar los contenidos o

13 En Wikipedia (n.d.) se señala “(...) Europeana es el portal del patrimonio cultural europeo que comenzó con dos millones de objetos digitales y cuya colección alcanzó los 29 millones de documentos en el año 2013" (...) teniendo como uno de sus objetivos "(...) agregar contenido cultural europeo para construir una fuente abierta y confiable del patrimonio europeo, representante de la diversidad cultural europea.

14 El Instituto de Ingenieros Eléctricos y Electrónicos (IEEE) (1990) define interoperabilidad como la habilidad de dos o más sistemas o componentes para intercambiar información y utilizar dicha información.

15 La cual señala que el acceso abierto brinda la posibilidad de que el usuario pueda leer, descargar, copiar, distribuir, imprimir; literatura erudita que se encuentre disponible de forma gratuita en internet (Budapest, 2002) 
metadatos de los mismos de forma automática en tanto esta es abierta y de acceso libre.

Implementar proyectos relacionados al patrimonio cultural que puedan conectarse y estar en concordancia con este ecosistema atravesado y construido por el código, la digitalización avanzada y los sistemas inteligentes, tienen condicionantes de orden cibernético (sistema de comunicación de las máquinas) donde los elementos acceso, reutilización e interoperabilidad son fundamentales para obtener cierta operatividad y efectividad o sustentabilidad para hablar en términos de smart city en su perspectiva tecnócrata.

\section{CONSIDERACIONES FINALES}

El desarrollo de las tecnologías de digitalización, y los softwares para gestión de objetos culturales y conformación de colecciones, su disponibilidad a un costo reducido y su facilidad de uso, fue determinante para cumplir los objetivos del proyecto propiciando el acceso y la apropiación, para motivar de alguna manera el ejercicio de la ciudadanía cultural y el desarrollo cultural general de la comunidad.

Las características del software utilizado que se abordaron, se consideraron fundamentales en la implementación de este tipo de proyecto si se pretende brindar acceso en los términos actuales, así como posibilitar los ciclos de reutilización de los datos y contenidos recogidos.

Analizando el acceso y la reutilización en los términos planteados, se entiende que tanto la visibilidad como el impacto de estos proyectos pueden presentar un condicionamiento desde el orden del código. A modo ilustrativo, este imperativo de orden cibernético (de las máquinas y sus sistemas de comunicación) se puede ejemplificar a partir de las siguientes situaciones: a) si un conjunto de datos no está estructurado en un esquema XML u otro lenguaje que permita la interoperabilidad, no solo no podrá ser accedido desde un móvil, sino que no podrá ser integrado a otras aplicaciones quedando fuera de ciclos de reutilización que dan valor agregado, o tomando nuestro caso b) si un repositorio digital no es realizado con un software que pueda trabajar con protocolo OAI-PMH Harvester no podrá ser recogido por otros agregadores o integrarse a los canales y flujos por donde circula y se hace visible la información cultural.

Las vinculaciones visibilizadas con respecto a las smart cities desde una perspectiva amplia son evidentes, el concepto de inteligente asociado a la ciudad implica la existencia no solo de sistemas de gestión y almacenamiento de información, sino una vinculación de la información con el espacio o territorio en donde ésta no solo representa dimensiones asociadas a la memoria y la cultura, sino que la conforman. También desde esta perspectiva podemos entender que un repositorio realizado bajo este tipo de software contribuye a la referida eficiencia en el uso de la información y los recursos asociados a la convivencia en un territorio, lo cual define a estas ciudades en el contexto de las TIC, en tanto permite la utilización y reutilización de la información por el mismo sistema de información generado como de los distintos actores vinculados al mismo en los distintos niveles de relacionamiento con la información y los espacios (productores, gestores y usuarios de esta información), además de permitir la reutilización por parte de otros sistemas. Como última consideración, se señala la relevancia que tomó configurar el proyecto como una experiencia interdisciplinaria donde se vincularon distintas áreas del conocimiento como son las ciencias de la información, la arquitectura y la ingeniería. En tal sentido, se considera necesario reproducir experiencias de este tipo.

\section{DEBATE}

El motivo del presente estudio no se limita a una descripción del proyecto a modo de compartir con la comunidad las políticas de metadatos, contenidos y acceso a los datos, así como la descripción de las distintas etapas transitadas en su implementación (lo cual tiene un gran valor a motivos prácticos), sino que pretende poner en discusión y ser un punto de partida para profundizar en la exploración de los alcances de estas herramientas y proyectos asociados al código abierto en el contexto de la digitalización, en tanto habilitan a múltiples comunidades mediante su accesibilidad y bajo costo a los procesos creativos para difundir, apropiarse y resignificar el patrimonio cultural, arquitectónico e histórico que las identifica.

Con estas ideas y exploraciones se busca motivar la reflexión sobre la dimensión cultural de las Smart Cities al introducir la figura del patrimonio y la cultura en relación a los proyectos de optimización de ciudades que encarna generalmente este concepto.

\section{REFERENCIAS}

Aguirre-Ligüera, N. y Ceretta, M. G. (2013). Construyendo un modelo de repositorio de acceso abierto para Uruguay. En: VI Encontro Ibérico EDICIC "Globalização, Ciência, Informação - Atas" (Porto, 4-6 nov. 2013). Porto: Universidade de Porto (pp. 1209-1219). Recuperado el 18 de noviembre de 2013, de: http://www.youblisher.com/p/745142VI-Encontro-Iberico-EDICIC-2013-Globalizacao-CienciaInformacao/

Barber, Gabriela; Lafluf, Marcos; "Interactive Projection Mapping: Proyecto Patrimonio ANGLO", p. 795-801 In: XX Congreso de la Sociedad Iberoamericana de Gráfica Digital. Blucher Design Proceedings, Volume 3, 2016, Pages 795-801, ISSN 2318-6968 DOI. http://dx.doi.org/10.1016/desprosigradi2016-627

Bonete Vizcancio, F. (2016) Smart Cities y patrimonio cultural. Una integración necesaria para el desarrollo en Revista Telos N.102, pag 3-7 Recuperado de https://telos.fundaciontelefonica.com

Bonete Vizcancio, F. (2016) "Smart City" y patrimonio cultural: las aplicaciones móviles de ciudades patrimonio de la humanidad españolas en Ciudad y Comunicación Pag.

Budapest Open Access Initiative (2002). La Iniciativa de Acceso Abierto de Budapest. GeoTrópico, 1(1), 98-100. Recuperado el 16 de julio de 2017 en: http://www.geotropico.org/1_1_Documentos_BOAl.html

Caldera Serrano, J. (2013). Metodología para el análisis de repositorio institucional de colecciones audiovisuales digitales. Documentación de las Ciencias de la Información, vol. 36, 209-219.

Caldera Serrano, J., Freire-Andino, R. O. (2016). Los metadatos asociados a la información audiovisual televisiva por "agentes externos" al servicio de documentación: validez, uso y posibilidades. Biblios, (62), 63-75. 
CEPAL. ONU. Santiago. Disponible en http://repositorio.cepal.org/bitstream/handle/11362/37089/S1 420540_es.pdf;jsessionid=C2F57B1C93C5E41C8D2FE7484 8DEC91E?sequence $=1$

Ceriotto, P. y Testa, P. (2011) LUCIS Directrices y recomendaciones para la aplicación de metadatos en repositorios institucionales. Versión preliminar. Recuperado el 14 de agosto de:http://bdigital.uncu.edu.ar/objetos_digitales/4123/directrice s-lucis-bd-2.pdf

De Giusti, M. et al. (2013) La Representación de Recursos en los Repositorios Institucionales. El Caso de estudio: SEDICI, Repositorio Institucional de la Universidad Nacional de La Plata., [Preprint]. Recuperado el 21 de agosto de 2013, de:.http://eprints.rclis.org/20341/

Europeana. (n.d.). En Wikipedia. Recuperado el 10 de marzo 2017 en https://es.wikipedia.org/wiki/Europeana

Heery, R. and Anderson, S. (2005). Digital Repositories Review, UKOLN and AHDS: 33.Recuperado el 02 de octubre de 2016 en: http://www.ukoln.ac.uk/repositories/publications/review200502/digital-repositories-review-2005.pdf

Institute of Electrical and Electronics Engineers (1990). IEEE Standard Computer Dictionary: A Compilation of IEEE Standard Computer Glossaries. New York.

Kucsma, Jason; Reiss, Kevin; Sidman, Angela (2010).“Using Omeka to Build Digital Collections: The METRO Case Study”. En: D-Lib Magazine, Vol. 16, n. 3-4. Disponible en: <http://www.dlib.org/dlib/march10/kucsma/03kucsma.htm>

Library of Congress Cataloging-inPublication Data Introduction to metadata. Spanish Introducción a los metadatos: vías a la información digital / editado por Murtha Baca; traducido al español por Marisol Jacas-Santoll. p. cm. ISBN 0-89236-535$8 \quad$ Disponible en http://d2aohiyo3d3idm.cloudfront.net/publications/virtuallibrar y/0892365358.pdf

Moreno Alonso, C. (2016).Desarrollo de un modelo de evaluación de ciudades basado en el concepto de ciudad inteligente (Smart city). Tesis (Doctoral), E.T.S.I. Caminos, Canales y Puertos (UPM).
Manovich, Lev (2003). El lenguaje de los nuevos medios de comunicación: la imagen en la era digital. Madrid, Editorial Paidós.

Manvoich, Lev (2008). Comprender los medios híbridos. Traducción de Eva Noriega y Melissa Mutchinick. Recuperado http://www.academia.edu/2800625/Comprender_los_ medios_h\%C3\%Adbridos

Omeka (2017). [Consulta: 15/02/2017]. Disponible en: http://omeka.org/

Patiño, J. A. (2014) Datos abiertos y ciudades inteligentes en América Latina. Estudio de cas.

Peset, Fernanda; Ferrerrer Sapena, Antonia; Subirats-Coll, Imma (2011). "Open data y Linked open data: su impacto en el área de bibliotecas y documentación”. En: El profesional de la información, Vol. 20, n. 2, pp. 165-173.

Pisarello, G. (2015 nov 19) Ciudad inteligente, para qué? El País.

Scheinfeldt, Tom (2010). Omeka and Its Peers. [Consulta: 15/10/2016]. Disponible en: https://omeka.org/blog/2010/09/21/omeka-and-peers/

Saorin, T. (2011) Catalogación de objetos culturales y difusión digital del patrimonio Anuario ThinkEPI, v. 5, p. 168-172.

Soarin, T. (2011) Exposiciones digitales y reutilización: aplicación del software libreOmeka para la publicación estructurada en MEI, II, Vol. 2, ํo 2, pág. 29. Disponible en: http://www.metodosdeinformacion.es/mei/index.php/mei/articl e/viewFile/IIMEI2-N2-029046/740

Solano, J. (2014). Smart Cultura y Open Data, conferencia dictada durante el ciclo Smart City: aproximación al desarrollo económico urbano del siglo XXI [en línea]. Universidad Complutense de Madrid y Banco Santander, 23 de julio.

Subirats J. (2015 nov 19). Ciudad inteligente, ciudad compartida. $\begin{array}{llll}\text { El país (de España) } & \text { Disponible en } \\ \text { http://ccaa.elpais.com/ccaa/2015/11/14/catalunya/144752595 }\end{array}$ 5 411073.html 